Research report

\title{
Recurrent perseveration correlates with abnormal repetitive locomotion in adult mink but is not reduced by environmental enrichment
}

\author{
Jamie A. Dallaire ${ }^{\mathrm{a}, *}$, Rebecca K. Meagher ${ }^{\mathrm{a}}$, María Díez-León ${ }^{\mathrm{a}}$, Joseph P. Garner ${ }^{\mathrm{b}}$, Georgia J. Mason ${ }^{\mathrm{a}}$ \\ a University of Guelph, Department of Animal and Poultry Science, 50 Stone Road East, Building \#70, Guelph, Ontario N1G 2W1, Canada \\ ${ }^{\mathrm{b}}$ Purdue University, Department of Animal Sciences, 125 South Russell Street, West Lafayette, IN 47907, USA
}

\section{A R T I C L E I N F O}

\section{Article history:}

Received 26 September 2010

Received in revised form 24 March 2011

Accepted 27 March 2011

Available online 2 April 2011

\section{Keywords:}

Recurrent perseveration

Impulsivity

Abnormal repetitive behaviour

Stereotypic behaviour

Environmental enrichment

American mink

\begin{abstract}
A B S T R A C T
We analysed the relationship between abnormal repetitive behaviour (ARB), the presence/absence of environmental enrichment, and two types of behavioural disinhibition in farmed American mink, Neovison vison. The first type, recurrent perseveration, the inappropriate repetition of already completed responses, was assessed using three indices of excessive response repetition and patterning in a biascorrected serial two-choice guessing task. The second type, disinhibition of prepotent responses to reward cues, a form of impulsivity, was tested in a locomotive detour task adapted from primate reaching tasks: subjects were required to walk around, rather than directly into, a transparent barrier behind which food was visible. In older adult females, recurrent perseveration positively predicted pre-feeding abnormal repetitive locomotion (ARL) in Non-enriched housing. High-ARL subjects also performed repeated (same-choice) responses more rapidly than low-ARL animals, even when statistically controlling for alternated (different-choice) response latency. Mink performed much less ARL following transfer to Enriched housing, but there was no corresponding change in recurrent perseveration. Thus, elevated recurrent perseveration is not sufficient for frequent ARL; and while captive environments do determine ARL frequency, in mink, they do not necessarily do so by modifying levels of perseveration. Disinhibition of prepotent responses to reward cues, meanwhile, did not predict ARL. In a separate sample of differentially housed young adults, neither type of behavioural disinhibition predicted ARL, and again, whether or not housing was enriched did not affect behavioural disinhibition despite affecting ARL. Thus, the relationship between recurrent perseveration and ARB may only develop with age; longitudinal studies are now required for confirmation.
\end{abstract}

(c) 2011 Elsevier B.V. All rights reserved.

\section{Introduction}

A range of activities termed 'abnormal repetitive behaviours' [1], 'restricted repetitive behaviours' [2], or 'stereotypic behaviours' $[3,4]$ spontaneously occur in certain human clinical conditions (e.g. mental retardation, schizophrenia, or autism) and in some caged animals. They include stereotypies, traditionally defined as repetitive, invariant, and apparently functionless [5,6], more morphologically variable activities such as hair/fur/feather plucking, and - in humans - behavioural expressions of obsessions or restricted interests. In this paper, we will use the term abnormal repetitive behaviour (ARB) to encompass all of these. ARB can be induced in research animals via extreme early maternal deprivation [7], treatment with psychostimulant drugs [8,9], or genetic manipulations, as in DAT knockout [10] and Fragile X models [11]. In both humans and manipulated research animals, altered functioning of the basal ganglia and corticostriatal circuits is implicated in ARB.

\footnotetext{
* Corresponding author. Tel.: +1 519824 4120x53557; fax: +1 5198369873.

E-mail address: j.ahloy.dallaire@gmail.com (J.A. Dallaire).
}

For example, in apomorphine/amphetamine/cocaine treated rats, drug-induced repetitive oral and whole-body movements correlate with overactivation of corticostriatal striosome-based circuits, relative to matrix-based circuits [12]; in deer mice, striatal infusion of dopamine receptor antagonists selectively decreases repetitive jumping, but not other motor activity [13]; conversely, DAT knockout induces repetitive locomotion and promotes dopaminergic activity in mice, especially in the striatum and nucleus accumbens [14,15].

The corticostriatal circuits or loops involved play an essential role in executive function and motor control [16], relaying information from processing areas of the cortex to the basal ganglia, where it is integrated and modulated, back to behaviour-generating cortical areas via the thalamus [17]. Each circuit contains both an inhibitory, "indirect" pathway and an excitatory, "direct" pathway [18]. Hypoactivity of the indirect relative to direct pathways is thought to lead to ARBs $[19,20]$. Statistical associations between perseveration - the tendency to inappropriately repeat actions [21] - and ARB provide evidence for this general impairment in behavioural inhibition. For example, in clinical human subjects participating in simple guessing or sequence-generation 
games, individuals who frequently repeated answers or short response sequences, despite instructions against doing so, also most frequently performed repetitive movements and had particularly circumscribed interests [22,23]. Perseveration has also been elicited in other circumstances known to cause ARB. For example, deprivation-reared rhesus monkeys performed more than twice as many nonrewarded lever presses as peer-reared controls in an operant extinction test [24]; and under high doses of amphetamine, rats trained to lever-press for food continued to press even after food had been delivered [25].

ARB is also performed by well over 85 million farm, zoo, companion, and laboratory animals worldwide (an estimate restricted to stereotypies), whose housing conditions and early experiences induce the behaviour $[19,26]$. Examples include pacing in zoo carnivores [27] and jumping and backward somersaulting in caged deer mice [28]. ARB is seldom, if ever, seen in wild, free-living animals, while in captivity, it is most prevalent and severe in housing conditions devoid of environmental enrichment $[26,29]$. Direct evidence that the ARB of Non-enriched captive animals reflects changes in corticostriatal function includes: haloperidol, which inhibits dopaminergic action in the nigrostriatal pathway, prevented captivity-induced jumping in bank voles [30]; and compared to Non-enriched and/or stereotypic deer mice, Enrichedreared individuals with negligible jumping/somersaulting showed elevated cytochrome oxidase activity in the motor cortex, striatum, and nucleus accumbens, elevated dendritic spine density in the motor cortex and striatum, and elevated brain-derived neurotrophic factor in the striatum [31-33]. Furthermore, behavioural evidence that captive animals' ARBs are part of a suite of changes inducing generalized disinhibition includes positive correlations between perseveration in operant tasks and ARB in a wide variety of captive species [1,34-41]. This mounting evidence is sufficiently compelling that two of us have advocated abandoning the traditional, purely descriptive definition of $A R B$, in favour of a mechanistic definition stipulating that ARB is the product of (among other causes) CNS dysfunction [1,3]: an issue we return to in the discussion.

Several gaps remain in our understanding of captive animals' $A R B$, however, three of which we focus on here. One concerns the nature and range of specific dysfunctions that underlie ARB in captive animals. There are multiple kinds of perseveration [21,42], which may correspond to ARBs with differing phenotypes $[1,41]$. Many animal experiments establishing a perseveration-ARB link have used some variant of operant extinction [34,37-40], which fails to distinguish between specific types of perseveration. Most exceptions have focused on "recurrent" perseveration, defined as the inappropriate repetition of an already completed response to a subsequent stimulus [42]. This type of perseveration correlated positively with repetitive locomotion/oral movements in caged birds [35,36], with bar-mouthing in C57BL/6J mice [41], but not in ICR CD-1 mice [43], and with repetitive movement in clinical human patients [22,23]. Other studies have tied "stuckin-set" perseveration, the inappropriate maintenance of a plan or goal, to more complex ARBs like barbering in mice $[1,41]$ and circumscribed interests in autistic humans [23]. Another proposed cause of ARB is failure to inhibit prepotent, emotionally motivated responses to reward cues, even when these responses are sub-optimal. This has also been termed affective perseveration [44], although as an 'impulsive' failure to inhibit initiation of behaviour, it arguably differs from 'compulsive' (perseverative) failures to inhibit repetition of behaviour [45]. The presence of repetitive, emotionally salient cues within an otherwise barren captive environment could conceivably lead to ARBs. Thus, for example, Cabib [46] argues that cage-climbing in food-deprived mice is elicited by the sustained presence of a food cue (the hopper).
A second unknown is the mechanism by which environmental enrichment reduces ARB. Environmental enrichment (EE) describes the provision of stimulation and opportunities to perform highly motivated behaviours [40,47]. EE is less successful at eliminating ARB than at preventing it in the first place $[48,49]$; in zoo animals, for example, adding EE typically only reduces time spent performing stereotypies by 50\% [47]. EE provided to animals who already perform ARB may only partially alleviate any underlying dysfunction, with more severely impaired animals being most resistant to enrichment. As an analogy, jumping/somersaulting in deer mice is most effectively reduced by adenosinergic $A_{1} / A_{2 A}$ receptor agonists in animals with already low levels of the behaviour [50].

The final unknown is whether previous associations between ARB and physiological, anatomical and behavioural indices of corticostriatal function reflect abnormal, dysfunctional changes induced by inadequate environments, or instead normal variation. In some instances this is clear: in deer mice, being raised with or without environmental enrichment induces group differences in jumping/somersaulting, perseveration and post-mortem indices of basal ganglia function [31,32,39]; while in African striped mice, wild-caught animals are less perseverative and show less frequent repetitive movements than captive-born individuals [51]. However, in many other instances, results are based on individual differences within similarly housed and treated subjects $[34,35,38,39,41]$. These individual differences could reflect normal variation, with a mean and variance no different from Enrichedhoused animals. For example, in adult humans who do not have obsessive-compulsive disorder, stuck-in-set perseveration has been found to correlate positively to obsessive-compulsivelike behaviours, despite these behaviours and perseveration both being in the normal, non-clinical, range [52].

Our study therefore had three main aims, each tested in a separate experiment. In Experiment 1, we tested the hypothesis that behavioural disinhibition underlies the performance of ARB in adult female American mink, Neovison vison, raised and housed in Non-enriched conditions. We predicted that recurrent perseveration and/or disinhibition of prepotent responses to reward cues would correlate positively with ARB. If recurrent perseveration proved predictive of ARB, we also expected subjects who frequently perform ARB to make repeated (same choice) responses, but not necessarily alternated (different choice) ones, particularly rapidly in the guessing task, as previously seen in parrots [36]. This would suggest that these individuals lack inhibitory control specifically over behavioural repetition, which may account for their ARB. In Experiment 2, we provided Experiment 1's subjects with environmental enrichment to test the hypotheses that EEs that reduce subjects' ARB also correspondingly reduce their perseveration/disinhibition, and that $\mathrm{EE}$ is least effective at reducing ARB in originally highly perseverative/disinhibited subjects. We predicted that both ARB and perseveration/disinhibition would be reduced, and that the smallest reductions in ARB would be observed in subjects with the highest levels of recurrent perseveration and/or disinhibition of prepotent responses to reward cues in Non-enriched housing. Finally, in Experiment 3, we screened differentially raised (Non-enriched vs. Enriched) young mink, to test the hypothesis that EE that prevents ARB from developing, as juveniles reach adulthood, also leads to the development of lower levels of recurrent perseveration and/or disinhibition of prepotent responses to reward cues. We predicted that mink in Enriched housing would show lower levels of both ARB and perseveration/disinhibition.

We chose American mink, N. vison, as a model because their ARB is very sensitive to cues predictive of food arrival, typically peaking in prevalence and rate immediately before the arrival of food $[53,54]$. This species is therefore a likely candidate for a relationship between ARB and disinhibition of responses to reward cues. 
Table 1

Ethogram of behavioural observations.

\begin{tabular}{|c|c|}
\hline $\begin{array}{l}\text { Abnormal repetitive } \\
\text { locomotion (ARL) }\end{array}$ & $\begin{array}{l}\text { Repetitive head/upper body movements (e.g. bobbing, } \\
\text { weaving, repeated either immediately or after a short } \\
\text { Scrabbling bout) or locomotive route-tracing (which } \\
\text { often includes performance of particular upper body } \\
\text { movements or of Scrabbling at fixed areas along the } \\
\text { route). } \\
\text { Unambiguous } A R L=3 \text { or more consecutive repetitions } \\
\text { Borderline } A R L=<3 \text { consecutive repetitions }\end{array}$ \\
\hline Scrabbling & $\begin{array}{l}\text { Subject scrabbles or scratches with forepaws against } \\
\text { the plastic walls of the cage or nestbox. If part of a } \\
\text { larger sequence of ARL, the behaviour is counted in } \\
\text { both categories. }\end{array}$ \\
\hline Normal Activity & $\begin{array}{l}\text { Any other activity (e.g. interacting with enrichment } \\
\text { items, walking, sniffing, grooming, eating, drinking, } \\
\text { urinating, defecating) }\end{array}$ \\
\hline Inactivity & $\begin{array}{l}\text { Subject is lying down motionless, except for minor } \\
\text { positional adjustments. }\end{array}$ \\
\hline
\end{tabular}

The main ARB of interest, in these mink, was abnormal repetitive locomotion (ARL: see Table 1), but we also considered another type of ARB, Scrabbling, separately for two reasons. First, unlike ARL, it often appears to be directed at other mink, and other authors have reported that similar "scratching" seems to be an attempt to enter an area to which access is denied [55]. Second, some authors consider such behaviour to be an ARB if it is repetitive enough [56], while others never do [57].

\section{Methods}

\subsection{Subjects}

For Experiments 1 and 2, mature adult females who performed at least some degree of ARB (two cohorts: 28 months old, $n=10 ; 52$ months old, $n=7$ ) were selected from Michigan State University's Experimental Fur Farm, where they had been raised and housed in typical fur farm wire mesh cages $(W 25 \mathrm{~cm} \times D 50 \mathrm{~cm} \times H 35 \mathrm{~cm})$ and moved in August 2009 to an indoor facility (see below). We chose to use relatively old animals because previous work has shown age to positively correlate with perseveration [37] and because ARB can be more difficult to eliminate using EE in older animals $[48,49]$. The only available mature adults were female ex-breeders, as male mink are typically killed in their first winter. For Experiment 3, we removed 4-month-old (juvenile) mink of both sexes from the same farm, and raised them in the same indoor facility. At this age young mink do not yet perform ARB [53,54]. Here, they were housed in either Non-enriched (NEE) or Enriched (EE) conditions (see below) for 7.5 months (into adulthood), before being tested. A total of 29 mink (NEE: 8F, 8M; EE: 7F, 6M) were used for behavioural observations in Experiment 3, although due to time constraints, only partially overlapping subsamples were tested for recurrent perseveration (NEE: 4F, 4M; EE: 3F, 3M) and disinhibition of prepotent responses to reward cues (NEE: 3F, 4M; EE: 3F, 2M).

\subsection{Housing and husbandry}

The indoor facility had an artificial light cycle yoked to sunrise and sunset times. Animals had ad lib. drinking water via a water line and nipple drinker, and were fed c. $150 \mathrm{~g}$ (females) or c. $250 \mathrm{~g}$ (males) of standard mink feed (a meat-based paste) once a day. Non-enriched housing consisted of a home cage $(W 60 \mathrm{~cm} \times D 75 \mathrm{~cm} \times H 45 \mathrm{~cm})$ with external nestbox $(W 21 \mathrm{~cm} \times D 25 \mathrm{~cm} \times H 30 \mathrm{~cm})$. In addition to the home cage, Enriched housing also included a second, larger cage $(W 120 \mathrm{~cm} \times D 75 \mathrm{~cm} \times H 45 \mathrm{~cm})$ containing a variety of manipulable objects, a plastic swing/hammock, and a $120 \mathrm{~cm}$ long trough of circulating filtered water $(5-10 \mathrm{~cm}$ deep), supplied because mink are semi-aquatic [58] and highly motivated to access water baths [59]. This Enriched cage was accessed from the home cage by using ramps to climb a tower ( $W 55 \mathrm{~cm} \times D 15 \mathrm{~cm} \times H 120 \mathrm{~cm})$, traversing a $3 \mathrm{~m}$ tunnel, and descending an identical tower with identical ramps. The older females were initially placed in Non-enriched housing for 4.5 months (for Experiment 1); but then transferred to Enriched housing for Experiment 2. Juveniles (Experiment 3) were pseudo-randomly assigned to either Non-enriched or Enriched housing immediately in August 2009, with sexes allocated equally between housing conditions.

\subsection{Behavioural observations}

For Experiment 1, we conducted behavioural observations on older adult females during three 6-day periods (September, October, and December); and for Experiment 2, during two 6-day periods after their transfer to Enriched housing (February and March). Subjects were given a new manipulable object on the afternoon preceding the start of each of the two Enriched observation periods. Animals were scanned every 9 min during the $3 \mathrm{~h}$ immediately preceding scheduled feeding. We used a modified type of instantaneous scan sampling, recording the behaviour being performed at the end of a 10-s observation period. This allowed us to gauge the repetitiveness of behaviours. Behaviours were classified according to the ethogram in Table 1. JAD conducted all observation periods but one (September), conducted by RKM. Observed frequencies were positively correlated between observers (e.g. $r=0.51, p=0.038$ for ARL) and thus were pooled for the remainder of our analyses. In Experiment 2, the observer was blind to mink identity and thence to levels of ARB in NEE. In Experiment 3, observations of young mink were conducted over 3 periods, by RKM (September and January) and by JAD (October).

\subsection{Testing for recurrent perseveration}

In humans, recurrent perseveration can be assessed from repetition of responses in guessing or sequencing tasks. For example, asked to guess the colour of successive playing cards drawn from a pack, or to place coloured markers in order, autistic children show a high degree of consecutive repetition (e.g. guessing 'black' several times in a row) and sequential dependency (i.e. patterning, or non-randomness) within series of responses [23,60-62]. A similar two-choice guessing task [22,63], also known as a "gambling" task, has been adapted for use with laboratory monkeys [64], birds [35,36], and mice $[41,43]$, and we used an analogous task for mink. Subjects chose between two doors (Fig. 1a) to receive either a food reward (either a piece of hotdog sausage, c. $0.75 \mathrm{~g}$, or a small dab of mink feed-switching on any trial after a subject declined to consume a reward), or a 30-s timeout. The "correct" (rewarded) door was randomly determined for each trial, but with a weighting designed to discourage prolonged repetition [36], using the formula: probability that left door is correct $=1-$ number of left responses in last 20 trials $/ 20$. Test order was randomized and counter-balanced across days. To maximize food motivation, mink were tested before their daily meal. Prior to the actual test, subjects underwent 'forced choice' training sessions in which one door or the other (in random order) was locked, and the other rewarded.

For Experiment 1, the older females were tested for recurrent perseveration in Non-enriched housing ( 2 days training, 6 days test, 20 trials per day) 3 months after the move from the farm. For Experiment 2, they were re-tested in Enriched housing ( 6 days test) during March 2010, after they had been living with enrichment for 11 weeks. Three subjects did not complete the guessing task during Experiment 2, leaving us with 14 subjects. For Experiment 3, young adults were tested (1 day training, 6 days test) during March 2010, after 7.5 months of differential housing. Training was shorter than for older females due to time constraints.

Three indices of recurrent perseveration were calculated, assessing either the amount of immediate repetition or the degree of sequential dependency in the sequence of responses:

(1) Excess Repetition: Standard score $(z)$ for observed number of responses which are immediate repetitions of the previous response vs. the number expected based on each subject's global side bias, calculated over all 120 trials, computed using the normal approximation to the binomial distribution. Positive scores indicate excessive repetition; negative scores, excessive alternation.

(2) Measures of sequential dependency:

(1) Third-order Markov Model: following Garner and colleagues [35,36,41], we compared the observed frequency of each of 16 possible 4-response sequences (e.g. RLRR or LRRL) to that expected based on each subject's global side bias. Thus, we obtained a $\chi^{2}$ value and calculated the corresponding $\mathrm{p}$ value. This value was then subtracted from 1 , yielding the probability that data are sequentially dependent, and logit transformed to make its distribution unbounded.

(2) Detrended Fluctuation Analysis: DFA is a fractal method of analyzing selfsimilarity in sequential binary data, recommended for ethological analyses of sequential behaviour $[65,66]$. DFA yields a single alpha score for each mink's response sequence, which equals 0.50 for random data sequences and increases with sequential dependency. Barring extreme cases, alpha scores for random sequences are unaffected by side bias.

We also tested whether average response latencies predict ARB, expecting that latencies to make repeated responses would negatively correlate to ARB, while alternated response latencies would not. We also tested whether repeated response latency negatively predicts ARB after controlling for alternated response latency, which would indicate that high-ARB individuals perform faster repeats than lowARB individuals with the same alternated response latency.

\subsection{Testing for disinhibition of prepotent responses to reward cues}

In the detour reaching task for primates, subjects must retrieve a toy or food reward behind a transparent barrier [67]. Failure to reach around, rather than directly into, the barrier is considered to reflect an impulsive lack of inhibitory control over behaviours influenced by appetitive stimuli $[68,69]$. In adapting the detour reaching task for mink, two main modifications were required. First, we attempted to ensure that our subjects, probably less reliant on visual cues than primates [58], also received olfactory cues of reward. Second, subjects were required to walk, rather than to reach, around a barrier. Similar tasks involving locomotive detours around 
A

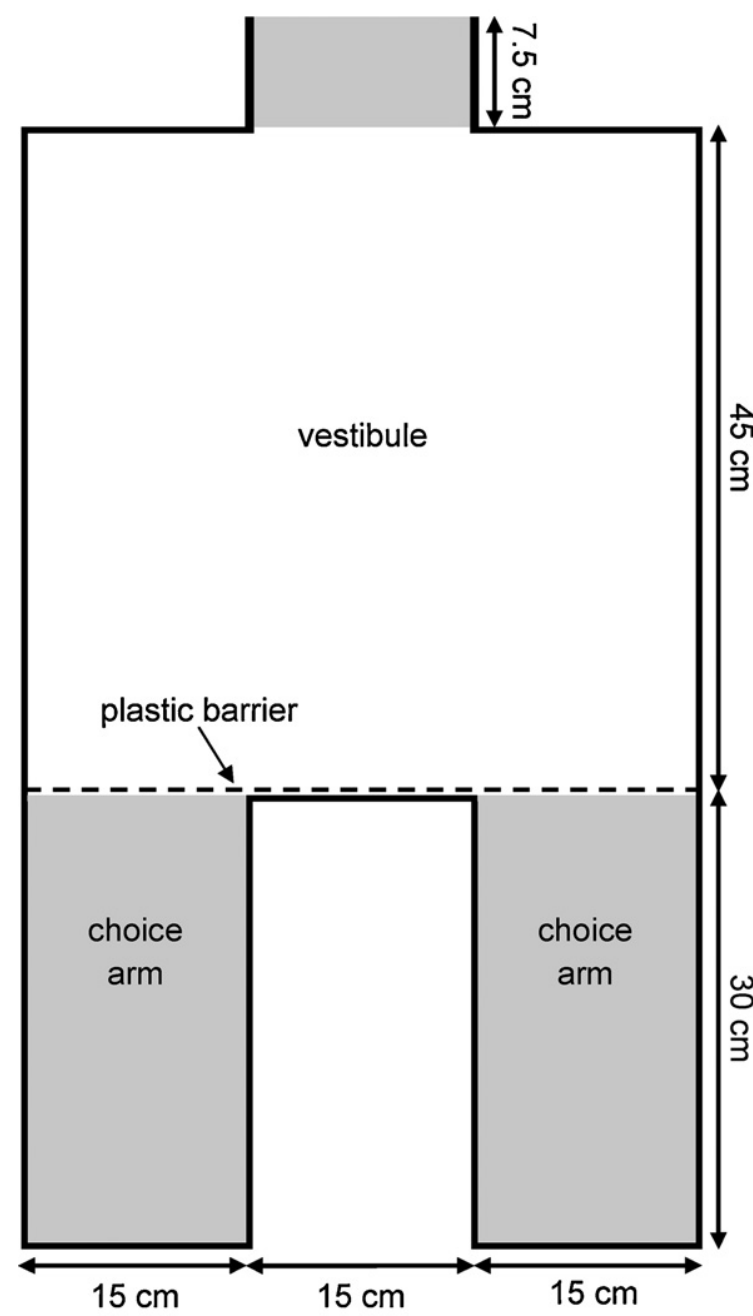

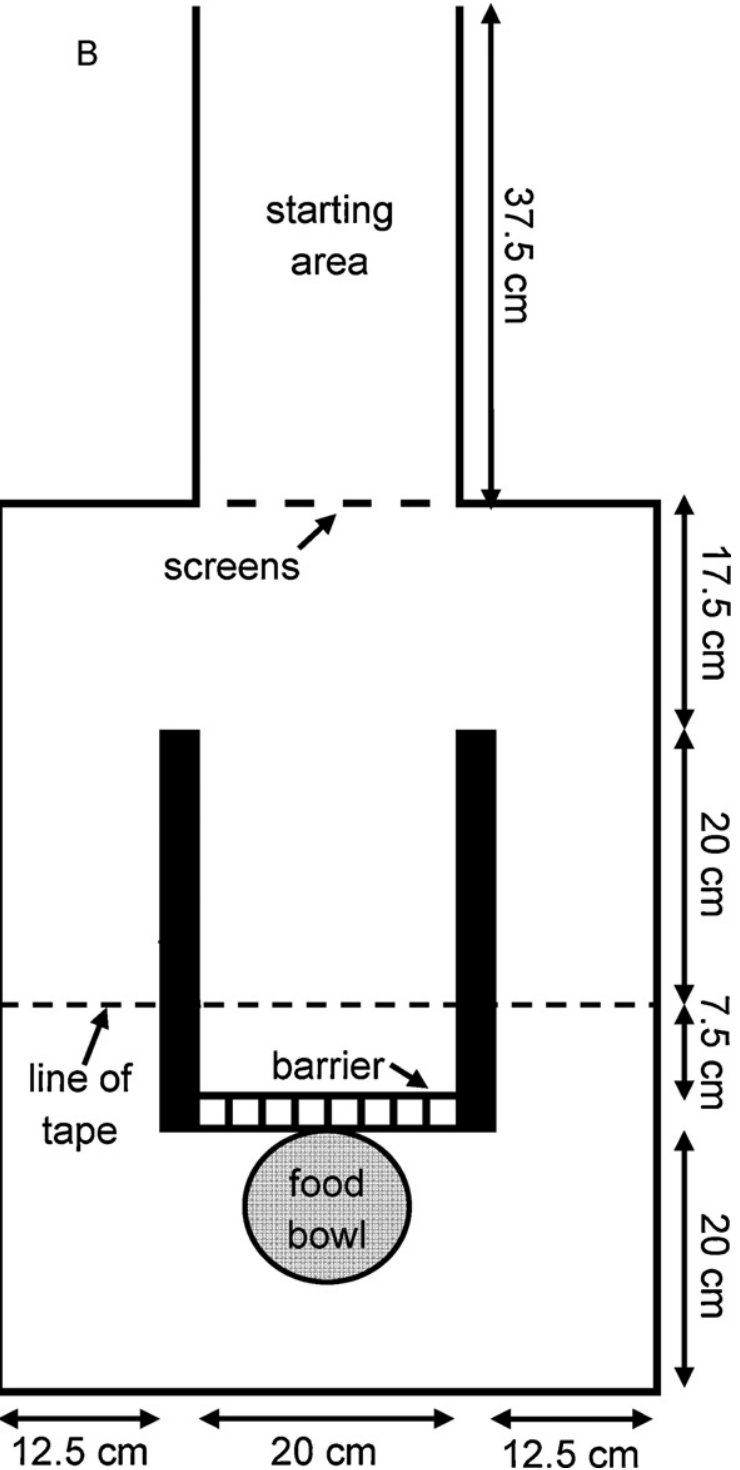

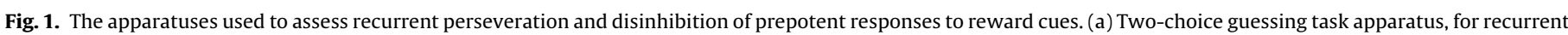

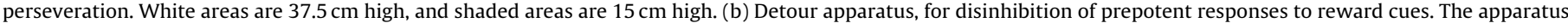
is $15 \mathrm{~cm}$ high along its entire length. The walls separating the three corridors are $2.5 \mathrm{~cm}$ wide.

transparent barriers have seen widespread use in non-primates [70], and have been used in human infants [71]. In our locomotive detour task, mink chose between three corridors within an apparatus (Fig. 1b). The middle corridor led to an impassable barrier, while the side corridors led to the area behind this barrier, where a red bowl was located, with a food reward (c. $0.75 \mathrm{~g}$ of hotdog sausage) on its lip. The barrier was made of two layers of metal mesh, between which an opaque plastic screen could be inserted. For Opaque trials, the screen was left in place, thus preventing all visual and, presumably, some olfactory food cues from reaching the mink. For Transparent trials, the screen was removed, making the bowl and the food reward visible through the mesh barrier. If mink fail to inhibit prepotent responses to reward cues, they should therefore enter the middle corridor more often on Transparent than on Opaque trials.

During testing, each subject participated in one 10-trial session daily. Food motivation was maximised as in the test for recurrent perseveration, and likewise, order of testing was randomized and counter-balanced across days. Each session contained 5 Opaque and 5 Transparent trials, in pseudo-random order, counterbalanced over 6 sessions. Disinhibition of prepotent responses to reward cues was quantified as the fraction of Transparent trials on which a subject responded in the middle corridor, statistically controlling for the fraction of Opaque trials which produced middle corridor responses.

For Experiment 1, older females were tested in the detour task for 6 sessions in November/December. Prior to testing, they were trained for 4 sessions (3 Opaqueonly, then 1 mixed). Early training sessions included only Opaque trials, to ensure that subjects could learn to perform the task in ideal conditions, without distracting cues of reward. Tamarins have been shown to perform a detour reaching task more proficiently if first trained with an opaque, rather than a transparent, barrier [44]. Because our detour task was a test of inhibitory control, it was crucial that subjects first have the opportunity to learn the correct response. Otherwise, they could not be expected to inhibit incorrect entries into the middle corridor, regardless of inhibitory control. Data were not collected in Experiment 2: older females were not retested in Enriched housing because increased experience performing detours around mesh within the tunnels of the EE housing represented a potential confounding explanation for any improvement in the task. Due to time constraints, young adults were trained only once in the ad lib. sausage consumption session, and then tested for 4 sessions in April 2010. Partially addressing the concern that EE subjects are more experienced with detours around mesh, the mesh barrier at the end of the middle corridor was removed, and either the opaque plastic screen or a clear piece of plastic with holes drilled into it (to allow passage of odours) was inserted, the latter for Transparent trials.

Older females (Experiment 1's subjects) also participated in subsequent trials to validate that middle responses in Transparent trials were due to failures to inhibit responses to the food cues, rather than to a lack of understanding that mesh is impassable, or failure to perceive the mesh from a distance. We ran 3 more sessions in which the food and bowl were not present in the apparatus. Food rewards were instead concealed outside the apparatus, held by the experimenter and delivered to subjects manually when they reached the bowl's former location. We then returned the bowl and food to the apparatus for 2 further sessions. If middle responses in Transparent trials were due to failures to inhibit responses to the food cues, then they should decline when the food and bowl were removed, and be reinstated when the food and bowl were returned. 


\subsection{Statistical analyses}

Markov Model sequential dependency scores and guessing task response latencies were log-transformed in order to normalize their distributions and to ensure that residuals computed from them in parametric tests would also be normally distributed. Using Minitab 15, General Linear Models (GLMs) were used for all analyses at $\alpha=0.05$ using Type II SS, except in repeated measures tests where Type III SS were used. Where the residuals of GLMs involving proportions (e.g. ARB time budgets) as predictive variables were non-normal, these tests were instead performed with the proportions arcsin-square root transformed, which normalized residuals. GLMs testing for a correlation between some indices of recurrent perseveration and arcsin-square root transformed ARB frequencies employed a square-root link function, because visual inspection revealed non-linear relationships. Cohort was included as a categorical factor in all analyses on older females, and sex in all analyses on young adults. To check robustness, tests involving ARL or Scrabbling were run twice, using either the behaviour's frequency as a fraction of the entire time budget or as a fraction of all activity. We also ran separate tests for Unambiguous and Borderline ARL, given that they were not necessarily expected to relate to disinhibition in the same way. Where change in ARL was the dependent variable, it was quantified in four different ways [49]: (a) absolute change in ARL frequency (EE-NEE), (b) relative change in ARL frequency (EE/NEE), (c) residual change in ARL frequency (residuals of a regression of ARL in EE on ARL in NEE), and (d) we also ran the GLM with ARL in EE as the dependent variable and ARL in NEE as a covariate, which is conceptually equivalent to residual analysis, but avoids problems associated with this approach [72,73]. When testing for the effects of recurrent perseveration, all three indices (see above) were tested separately. When testing for the effects of disinhibition of prepotent responses to reward cues, the fraction of middle responses in Transparent trials was corrected for the fraction of middle responses in Opaque trials. To conserve degrees of freedom, where models included covariates other than our main variable of interest, the only interactions included were those between cohort (or sex) and the latter. We did, however, verify that including further interactions made no qualitative difference to our results. To test for effects of housing on the frequency of different behaviours in older females, we used a repeated measures GLM with subject (nested within cohort) as a random factor. This procedure was also used to investigate the effect of trial type and testing period on the number of middle responses in the detour task and, in this case, followed up with orthogonal paired $t$-tests comparing trial types within each testing period.

\section{Results}

Effects of cohort, sex, and interactions are non-significant unless stated otherwise. All scatter plots show partial regressions: ARL frequency and the independent variable of interest were separately regressed against all other independent variables in the model, and the residuals obtained from these regressions were then corrected for range (by adding the variable's mean) and plotted against each other.

\subsection{Experiment 1 -perseveration/disinhibition and ARB in Non-enriched conditions}

All three indices of recurrent perseveration significantly intercorrelated in the expected direction (all correlations: $F_{1,13}>12.82$, $p<0.003$ ). All indices of high recurrent perseveration positively correlated with the frequency of Unambiguous ARL, though this was only a trend for DFA (Fig. 2a-c). Depending on the index used, recurrent perseveration explains $22-37 \%$ of the variance in Unambiguous ARL. No index significantly predicted the frequency of Borderline ARL (all indices: $F_{1,13}<0.32, p>0.579$ ), nor the frequency of Scrabbling (all indices: $F_{1,13}<0.32, p>0.582$ ).

Average response latency negatively correlated with Unambiguous ARL both for repeated $\left(F_{1,13}=16.98, p=0.001\right)$ and alternated responses $\left(F_{1,13}=4.86, p=0.046\right)$. There was a trend toward an interaction between repeated response latency and cohort $\left(F_{1,13}=3.75, p=0.075\right)$, due to a difference in slopes between the cohorts, with that of the younger group being steeper. Average repeat latency negatively predicted ARL even when controlling for average alternation latency (Fig. 3 ).

For disinhibition of prepotent responses to reward cues, our validation trials showed that responses into the middle corridor (into the barrier) on Transparent trials were increased by food-related cues, as predicted (Fig. 4). There was a significantly higher mean
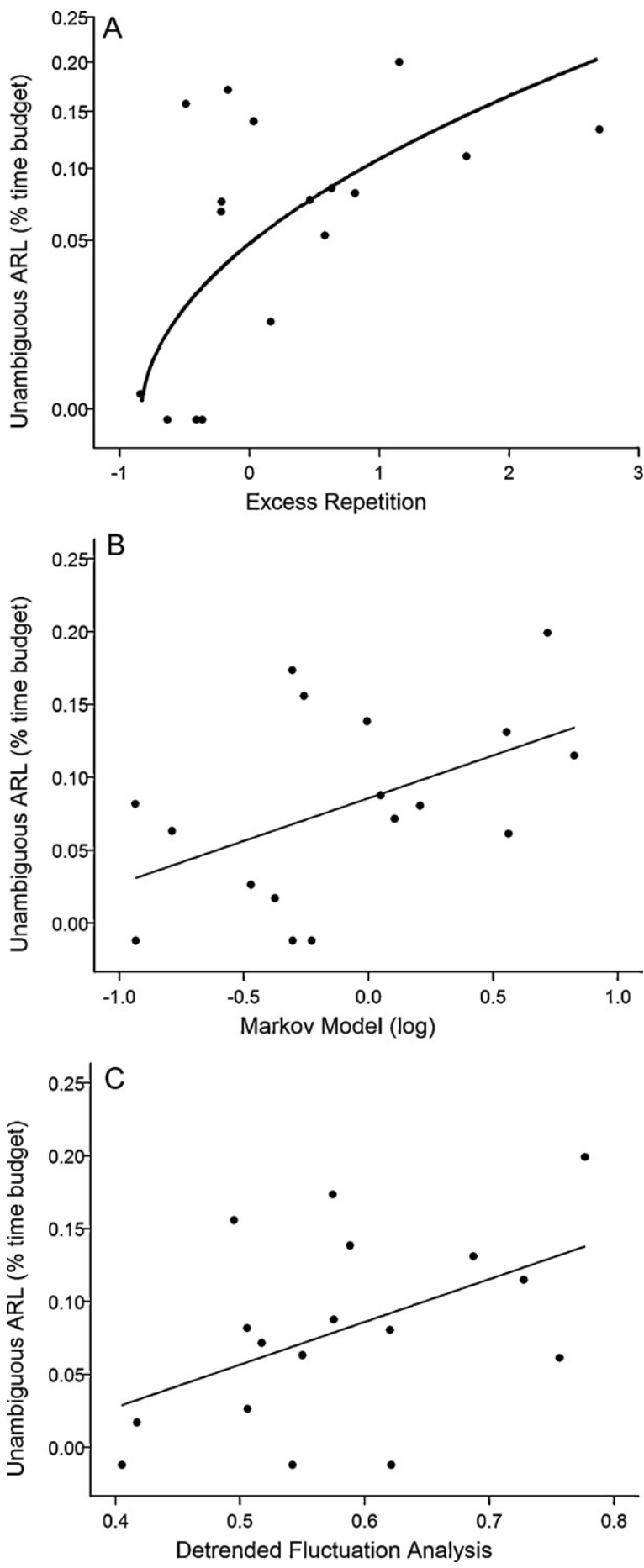

Fig. 2. Recurrent perseveration positively predicted the frequency of Unambiguous ARL. This was a significant relationship for (a) Excess Repetition $\left(F_{1,13}=7.91\right.$, $p=0.015)$ and (b) Markov Model $\left(F_{1,13}=4.73, p=0.049\right)$, but was only a trend for (c) $\operatorname{DFA}\left(F_{1,13}=4.19, p=0.061\right)$.

fraction of middle responses in Transparent trials than in Opaque trials overall $\left(F_{1,79}=15.58, p<0.001\right)$. Planned comparisons (paired $t$-tests) showed that this difference was present in the first testing period $(t=5.55, p<0.001)$, but not in the testing period without food cues $(t=0.57, p=0.579)$, and was reinstated when food cues were returned $(t=2.60, p=0.019)$. There was also an overall effect of cohort (but no interaction with trial type), indicating that the younger cohort produced more middle responses, regardless of trial type $\left(F_{1,79}=45.53, p<0.000\right)$, and the effect of subject was 


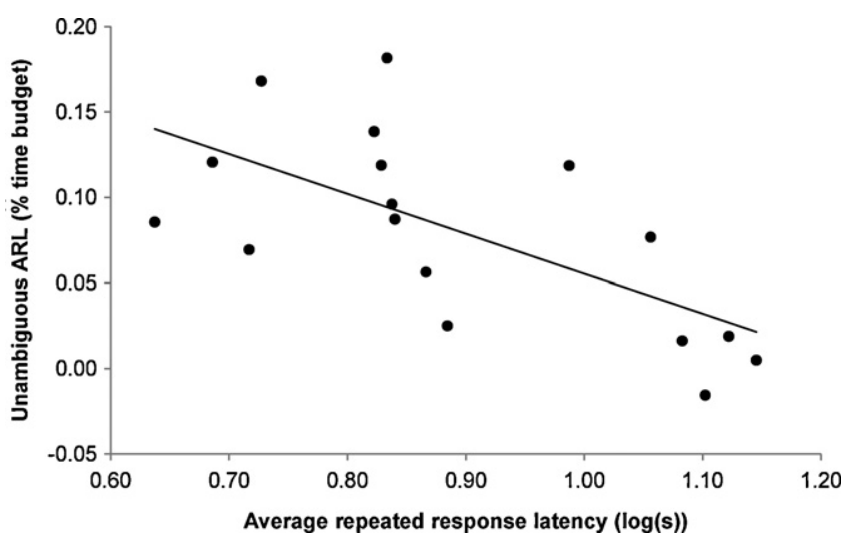

Fig. 3. Unambiguous ARL frequency negatively correlates with repeated response latency, controlled for alternated response latency $\left(F_{1,12}=10.40, p=0.007\right)$.

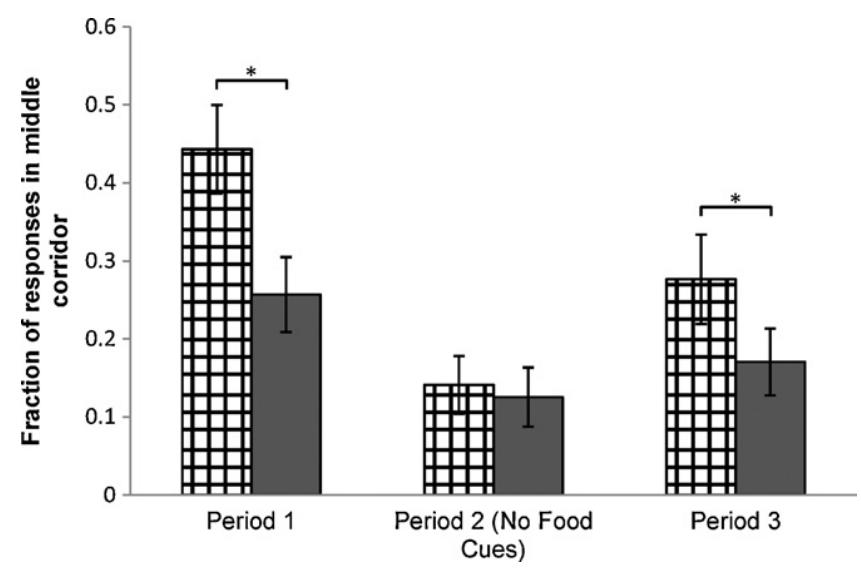

Fig. 4. The test for disinhibition of prepotent responses to reward cues was successfully validated. Subjects entered the middle corridor more often (mean $\pm \mathrm{SE}$ ) in Transparent trials (grid) than in Opaque trials (shaded) during Periods 1 and 3, but not during Period 2 when food cues were absent.

significant $\left(F_{15,79}=7.73, p<0.001\right)$, indicating that individual mink were consistent across testing period and trial type. Disinhibition of prepotent responses to reward cues did not significantly predict any index of recurrent perseveration (all indices: $F_{1,12}<0.85$, $p>0.374$ ), suggesting no underlying commonalities. Disinhibition of prepotent responses to reward cues also did not correlate with Unambiguous ARL (Fig. 5), Borderline ARL $\left(F_{1,12}=0.06, p=0.804\right)$, or Scrabbling $\left(F_{1,12}=0.89, p=0.364\right)$.

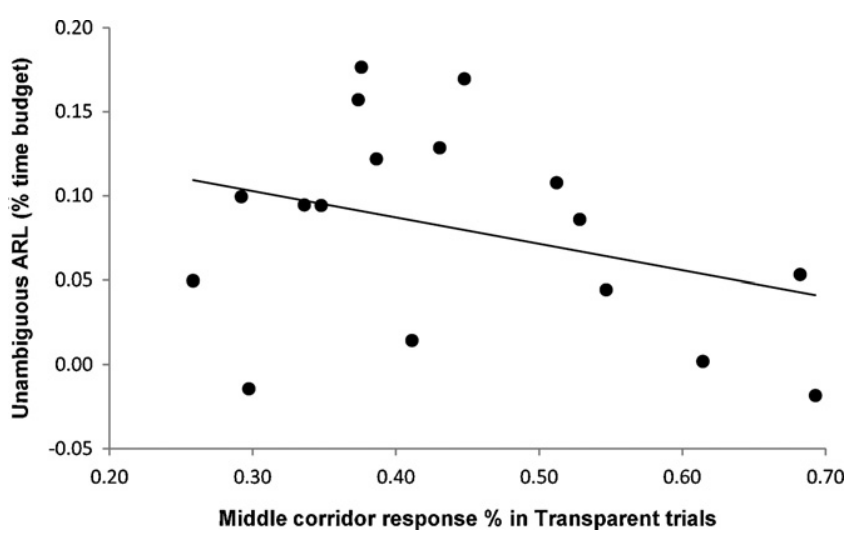

Fig. 5. Disinhibition of prepotent responses to reward cues does not predict the frequency of Unambiguous ARL $\left(F_{1,12}=0.06, p=0.809\right)$.
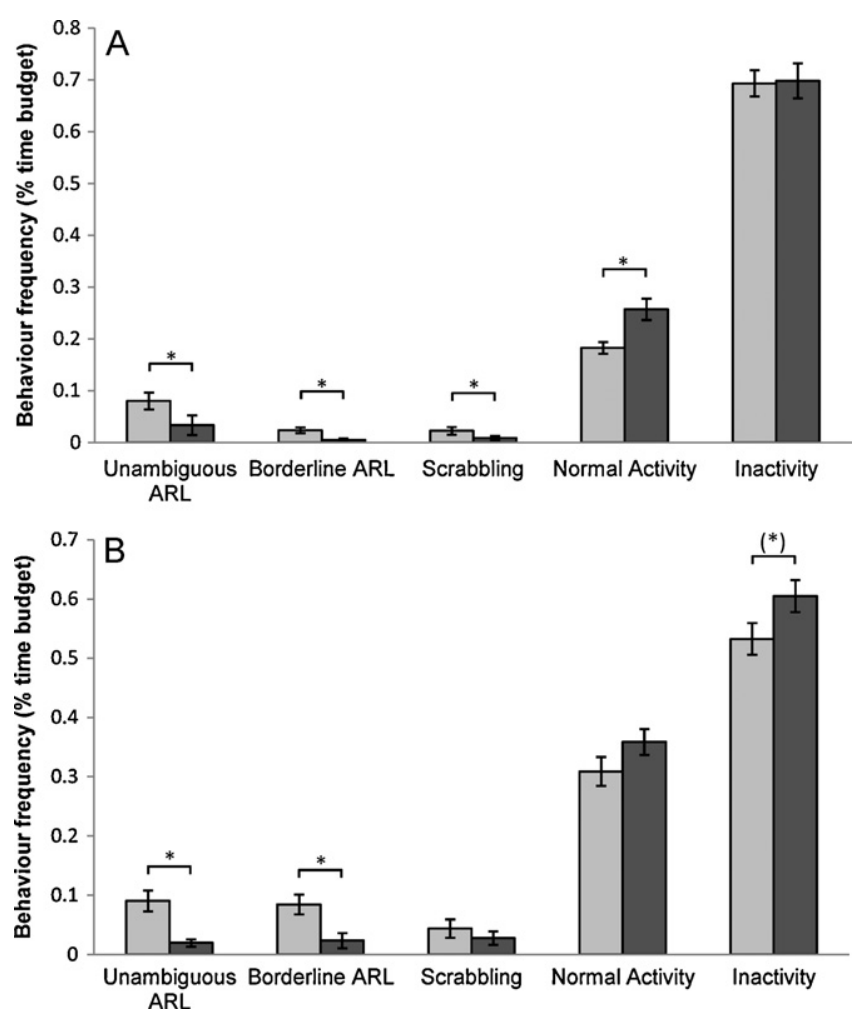

Fig. 6. Mean frequency of behaviours $( \pm S E)$ as proportion of entire pre-feeding time budget (light bars: Non-enriched housing; dark bars: Enriched housing) in (a) older females and (b) differentially housed young adults. The sum of behaviour frequencies in each housing treatment exceeds $100 \%$ because the two ARL categories partially overlap with Scrabbling.

\subsection{Experiment 2-environmental enrichment, perseveration/disinhibition and $A R B$}

Following transfer to Enriched housing (Fig. 6a), older female subjects performed significantly less Unambiguous ARL $\left(F_{1,15}=5.73, p=0.030\right)$, Borderline ARL $\left(F_{1,15}=10.94, p=0.005\right)$, and Scrabbling $\left(F_{1,15}=7.10, p=0.018\right)$. They performed more Normal Activity $\left(F_{1,15}=8.17, p=0.012\right)$, and there was no difference in Inactivity $\left(F_{1,15}=0.02, p=0.891\right)$. Changes in Unambiguous ARL ranged from a $25 \%$ decrease in terms of an animal's entire prefeeding time budget (which represented the complete elimination of the behaviour) to a $12 \%$ increase (a near doubling). No index of recurrent perseveration, as assessed in Experiment 1, predicted the change in Unambiguous ARL between Non-enriched and Enriched housing, whether in terms of absolute change (all indices: $F_{1,13}<0.70, p>0.419$ ), relative change (all indices: $F_{1,9}<0.40$, $p>0.541$ ), residual change (all indices: $F_{1,13}<0.40, p>0.539$ ), or as assessed in General Linear Models which control for ARL in NEE (all indices: $F_{1,12}<0.71, p>0.417$ ). Disinhibition of prepotent responses to reward cues also did not predict change in Unambiguous ARL (absolute: $F_{1,12}=0.33, p=0.586$; relative: $F_{1,8}=0.50, p=0.503$; $F_{1,12}=0.17, p=0.684$; residual: GLM: $\left.F_{1,11}=0.39, p=0.544\right)$.

Data on recurrent perseveration, collected at the end of Experiment 2, showed that despite the great decrease in ARL, recurrent perseveration did not decrease after transfer to Enriched housing, but was instead slightly, non-significantly higher according to most indices (all indices: $F_{1,12}<0.68, p>0.427$ ). Equivalence tests showed that we had sufficient power to exclude, at $\alpha=0.05$, a decrease in recurrent perseveration equivalent to that expected based on the observed decrease in Unambiguous ARL (extrapolated from Fig. 2a-c) for all indices. When reassessed in Experiment 2 , all indices of recurrent perseveration were positively inter- 
correlated, though only 2 of these 3 correlations were significant (Excess Repetition and other indices: $F_{1,10}>10.34, p<0.009$; DFA and Markov Model: $F_{1,10}=3.95, p=0.075$ ). Only one of these indices predicted the frequency of Unambiguous ARL in Enriched housing (DFA: $F_{1,10}=6.99, p=0.025$; Excess Repetition: $F_{1,10}=1.56$, $p=0.240$; Markov Model: $F_{1,10}=0.54, p=0.478$ ). The amounts of change in ARL and of change in recurrent perseveration, after mink were given environmental enrichment, were positively correlated, but unlike in previous research on songbirds [35], this relationship was not significant (all indices: $F_{1,10}<2.04, p=0.184$ ). No index of recurrent perseveration in EE significantly predicted Borderline ARL (all indices: $F_{1,10}<2.21, p>0.168$ ) nor Scrabbling (all indices: $\left.F_{1,10}<0.48, p>0.506\right)$.

\subsection{Experiment 3-does Enriched rearing affect perseveration?}

Enriched-housed juveniles/young adults performed significantly less Unambiguous ARL $\left(F_{1,25}=16.77, p<0.001\right)$ and Borderline ARL $\left(F_{1,25}=15.30, p<0.001\right)$, and tended to devote more time to Inactivity $\left(F_{1,25}=3.43, p=0.076\right)$, than did their Nonenriched counterparts (Fig. 6b). Housing did not significantly affect Scrabbling $\left(F_{1,25}=0.83, p=0.371\right)$ or Normal Activity $\left(F_{1,25}=2.07\right.$, $p=0.163$ ). Females performed more Borderline ARL than did males $\left(F_{1,25}=4.38, p=0.047\right)$. Results presented here are for the entire juvenile sample. When we separately analysed only the subsamples which were used for tests of recurrent perseveration or of disinhibition of prepotent responses to reward cues, the effects of EE on both categories of ARL remained significant, but the trend for Inactivity disappeared. There was no main effect of housing on any index of recurrent perseveration (all indices: $F_{1,10}<2.88, p>0.121$ ). On some indices, females showed higher recurrent perseveration than males (Excess Repetition: $F_{1,10}=7.48, p=0.021$; DFA: $\left.F_{1,10}=6.68, p=0.027\right)$. There was also a significant sex $\times$ housing interaction for the Markov Model index $\left(F_{1,10}=32.61, p<0.001\right)$, indicating that Non-enriched males were more perseverative than Enriched males ( $t=5.06, p=0.004$ ), but Non-enriched females were less perseverative than Enriched females $(t=3.77, p=0.013)$. Housing did not have any effect on disinhibition of prepotent responses to reward cues in these young adults $\left(F_{1,7}=0.02, p=0.888\right)$.

Recurrent perseveration in young adults predicted neither Unambiguous ARL (all indices: $F_{1,9}<2.44, p>0.157$ ) nor Borderline ARL (all indices: $F_{1,9}<2.69, p>0.140$ ). DFA was the only index for which there was any semblance of a positive relationship with Unambiguous ARL. Equivalence tests on the slopes of perseveration-ARL relationships showed, however, that for all indices, we had insufficient power to exclude, at $\alpha=0.05$, a positive relationship with a slope equivalent to the lower bound of the $95 \%$ confidence interval of the significant slope observed in older females. Disinhibition of prepotent responses to reward cues in young adults predicted neither Unambiguous ARL $\left(F_{1,6}=0.03\right.$, $p=0.873)$ nor Borderline ARL $\left(F_{1,6}=0.82, p=0.400\right)$.

\section{Discussion}

Our first aim, in Experiment 1, was to screen for recurrent perseveration and disinhibition of prepotent responses to reward cues in the same animals, and to assess their relationships with ARB induced by long-term housing in Non-enriched conditions. Our modification of the detour reaching task is the first application of this type of technique to a non-primate for the purpose of assessing disinhibition of prepotent responses to reward cues. We found no relationship between ARB and disinhibition of prepotent responses to reward cues, even though mink ARB is stimulated by cues predictive of food and by food restriction [53,54]. This might reflect a problem with the test itself: future work could further validate this as a test for responsiveness to appetitive cues in non-primates by manipulating cue strengths (c.f. [74]) or by testing with a wider array of reward types. A methodological improvement might be to train subjects more extensively before testing, to eliminate middle responses in Opaque trials, leaving only those responses in Transparent trials that actually stem from inhibitory failure. Non-primate experiments involving detours around transparent barriers have tended to focus not on impulsive disinhibition, but rather on "insight" or social learning [75,76] (for a recent discussion which bridges this gap, see [77]). Thus, while many species more readily perform detours around opaque than around transparent barriers $[74,78,79]$, they may do so simply because of poor comprehension. However, one of these studies [74] suggested that the difficulty of the task was affected by motivational characteristics of the goal behind the transparent barrier. This parallels findings from our validation trials: only when food cues were available behind the blind-ended middle section did subjects move directly into it, toward the food reward, more often when the barrier was transparent than when it was opaque. When food cues were absent, in contrast, the barrier being transparent had no such effect. Thus, our test as conducted does seem valid. We also found that individual mink were stable in their degrees of disinhibition in this task (Fig. 4), suggesting reliability as well as validity. The lack of a relationship between pre-feeding ARB and disinhibition of prepotent responses to reward cues could thus reflect a genuine lack of effect. Therefore, individual differences in pre-feeding ARB in mink likely do not reflect behaviour that is readily controlled by reward cues. Previous work has shown, however, that different aspects of impulsive behaviour can be dissociated from each other [80]. It is possible, then, that performance on tests designed to measure other impulsive deficits, like delay discounting or premature responding, would correlate with ARB, even though performance on the detour task did not. Premature responding may be particularly likely to correlate with ARB in mink, given that high-ARB individuals responded with the shortest latencies in the guessing task for recurrent perseveration. Within the context of that experiment, however, these responses were simply fast, not premature or inappropriate, and should not be taken as measures of impulsivity.

Recurrent perseveration successfully explained $22-37 \%$ of the variance in Unambiguous ARL in our older subjects, depending on the index used. These results, and similar ones obtained after transferring the mink to Enriched housing, provide further support for a widespread relationship between recurrent perseveration and ARB, particularly stereotypy (of which ARL is one type) [22,23,35,36,41]. We used one measure specifically sensitive to repeated responses, and two sensitive to higher order patterns. Furthermore, our prediction that older females with frequent ARL would perform repeated responses with shorter latencies than would lower ARL subjects was supported, paralleling existing data from birds [36]. While this was also true of alternated responses, further tests showed that even when alternated response latency is controlled for, high-ARL subjects perform repeated responses with particularly short latencies. This provides further evidence for the idea that disinhibition, particularly that of repeated behavioural responses, is mechanistically involved in recurrent perseveration.

Our data also revealed some exceptions to the positive relationship between pre-feeding ARB and recurrent perseveration. First, recurrent perseveration was found not to predict Borderline ARL, behaviours that were not quite repetitive enough to meet our criterion for Unambiguous ARL: a discrepancy that further suggests that recurrent perseveration is involved specifically in the repetitive aspect of abnormal repetitive behaviour. Second, despite its very repetitive appearance, 'Scrabbling', in which mink rapidly scratch at a plastic surface with their forepaws, did not co-vary with recurrent perseveration in either housing condition; thus the relationship between ARB and perseveration is not a universal one (for other 
recent exceptions in mice, see $[40,43])$. Finally, when this relationship was re-examined in young adult mink in Experiment 3, no correlation between recurrent perseveration and any type of ARB existed in this group. Statistical power was inadequate for this test in our young adults, however. If there truly was no relationship in younger mink, the relationship between ARB and recurrent perseveration may be one that develops over time. A longitudinal study is now needed, both to properly replicate the lack of a relationship in younger mink, and to formally investigate whether recurrent perseveration only comes to be involved in the control of ARB in older animals.

Our study did not address the involvement of the basal ganglia and corticostriatal circuits in behavioural disinhibition and ARB. Neuro-anatomically, there are multiple parallel corticostriatal circuits responsible for different aspects of behavioural control [81]. Disinhibition in different circuits may result in perseveration at different steps of behavioural control, and in ARBs of corresponding types. For example, Garner [1] has speculated that both recurrent perseveration and stereotypies are repetitive manifestations of dorsal striatal (putamen), sensorimotor circuit disinhibition. While selective neuropharmacological motor circuit manipulations can induce ARB [13,82], future work is needed to show specific involvement of the motor circuit in recurrent perseveration. Disinhibition of prepotent responses to reward cues, meanwhile, can result from altered functioning of the ventral striatal, limbic circuit (ventral striatum and associated areas, principally the nucleus accumbens shell) [83]. For example, neuropharmacological manipulation of rats' nucleus accumbens led to preferences for small, immediate rewards over large, delayed ones [84]. Social isolation caused the same effect in rats [85], in addition to altering levels of dopamine transporter protein in the nucleus accumbens [86]. Limbic function may be tied to captive animals' ARBs: for example, crib-biting horses had higher nucleus accumbens dopamine receptor densities than did non-crib-biters [87], and the frequency of pigs' spontaneous chain-chewing, before amphetamine administration, correlated positively with subsequent amphetamine-induced locomotion [88], an accumbens-mediated response [89]. Future research should attempt to directly validate our guessing and detour tasks as measures of, respectively, dorsal and ventral striatal function. This could be done by assessing performance on these tests after selective basal ganglia lesioning or administration of dopaminergic agents. The frozen brains of the animals used in our experiments could also be tested, via cytochrome oxidase staining, for a correlation between guessing or detour task performance and activity in corresponding striatal structures.

Experiment 2 tested the hypotheses that highly perseverative animals would have ARB particularly resistant to reduction by EE, and that EE that effectively reduced ARB would also correspondingly reduce perseveration. Neither of these hypotheses was supported. First, recurrent perseveration did not predict how easy ARB was to eliminate. Given the great range in changes in all types of ARB following transfer to Enriched housing, this lack of effect could not reflect ceiling or floor effects. Instead this suggests that, at least in mink, factors aside from processes affecting behavioural inhibition are responsible for EE effectiveness. Thus even though Experiment 1 showed that recurrent perseveration closely predicted the time-budget devoted to ARL, it did not predict another index of severity in the same subjects: resistance to enrichment. One possible explanation relates to how much time individuals devote to the new behavioural opportunities offered by enrichments, and/or how rewarding they find them $[40,49]$. We found no support for the second hypothesis tested in Experiment 2: even though after 2-3 months of EE exposure, ARL was reduced on average by over half, recurrent perseveration was not reduced at all. This is unlikely to simply be a Type II error, as equivalence tests suggested we had sufficient power. Thus, providing enrichment to Non-enriched raised mink did not reduce perseveration concomitantly as it reduced ARB. Our result shows that changes in recurrent perseveration are clearly not necessary conditions for changes in ARB, and acts as a further reminder that processes relating to perseveration are not the only mechanisms underlying ARB performance.

In Experiment 3, we screened differentially housed (Nonenriched vs. Enriched) young mink, to test for the hypothesis that EE that prevents ARB from developing as juveniles reach adulthood, also leads to the development of lower levels of perseveration. Unlike in older adults, we found no relationship between ARL and recurrent perseveration. Equivalence tests, however, showed that power was insufficient to reach a firm conclusion. It is plausible, then, that the lack of such a relationship in young adults reflects a Type II error, rather than true lack of effect. Mirroring our findings from Experiment 2, we found no difference in recurrent perseveration between Enriched and Non-enriched subjects, despite again a large difference in ARB. Furthermore, neither was there any difference in disinhibition of prepotent responses to reward cues. Again, in younger mink, but not in older females, this test had relatively low power. That mink were no less perseverative/disinhibited in Enriched than in Non-enriched housing suggests that perseveration per se is not necessarily abnormal, and does not have to reach a dysfunctional range of severity in order to be involved in the causation of ARB, at least in older adults. Caution is warranted, however, because we lack a baseline level of perseveration (e.g. from wild or feral mink) to which we can compare the values we obtained. We may also have missed a critical period for enrichment to affect perseveration-our differentially housed young adults had been kept in Non-enriched housing until they were 3 months old. Future work may address this by enriching animals from birth (e.g. in mink: Díez-León, in preparation) or by comparing captive-born mink to wild-caught individuals [51].

\section{Acknowledgments}

We thank Angelo Napolitano and the Michigan State University Experimental Fur Farm staff. Funding was provided by an NSERC Discovery grant to GJM.

\section{References}

[1] Garner JP. Perseveration and stereotypy-systems-level insights from clinical psychology. In: Mason G, Rushen J, editors. Stereotypic animal behaviour: fundamentals and applications to welfare. Wallingford, England: CABI; 2006. p. 121-52.

[2] Lewis M, Kim SJ. The pathophysiology of restricted repetitive behavior. J Neurodev Disord 2009;1:114-32.

[3] Mason G. Stereotypic behaviour in captive animals: fundamentals and implications for welfare and beyond. In: Mason G, Rushen J, editors. Stereotypic animal behaviour: fundamentals and applications to welfare. Wallingford, England: CABI; 2006. p. 325-56.

[4] Mills D, Luescher A. Veterinary and pharmacological approaches to abnormal repetitive behaviour. In: Mason G, Rushen J, editors. Stereotypic animal behaviour: fundamentals and applications to welfare. Wallingford, England: CABI; 2006. p. 286-324.

[5] Odberg F. Abnormal behaviours: stereotypies. In: Garsi J, editor. Proceedings of the 1 st world congress on ethology applied to zootechnics. Madrid: Industrias Graficas Espana; 1978. p. 475-80.

[6] Mason GJ. Stereotypies: a critical review. Anim Behav 1991;41:1015-37.

[7] Cross HA, Harlow HF. Prolonged and progressive effects of partial isolation on the behaviour of Macaque monkeys. J Exp Res Pers 1965;1:39-49.

[8] Ernst AM, Smelik PG. Site of action of dopamine and apomorphine on compulsive gnawing behaviour in rats. Cell Mol Life Sci 1966;22:837-8.

[9] Sahakian BJ, Robbins TW, Morgan MJ, Iversen SD. The effects of psychomotor stimulants on stereotypy and locomotor activity in socially deprived and control rats. Brain Res 1975;84:195-205.

[10] Gainetdinov RR, Wetsel WC, Jones SR, Levin ED, Jaber M, Caron MG. Role of serotonin in the paradoxical calming effect of psychostimulants on hyperactivity. Science 1999;283:397-401.

[11] Hayashi ML, Rao BSS, Seo JS, Choi HS, Dolan BM, Choi SY, et al. Inhibition of p21-activated kinase rescues symptoms of fragile $X$ syndrome in mice. Proc Natl Acad Sci USA 2007;104:11489-94 
[12] Canales JJ, Graybiel AM. A measure of striatal function predicts motor stereotypy. Nat Neurosci 2000;3:377-83.

[13] Presti MF, Mikes HM, Lewis MH. Selective blockade of spontaneous motor stereotypy via intrastriatal pharmacological manipulation. Pharmacol Biochem Behav 2003;74:833-9.

[14] Ralph RJ, Paulus MP, Fumagalli F, Caron MG, Geyer MA. Prepulse inhibition deficits and perseverative motor patterns in dopamine transporter knockout mice: differential effects of D1 and D2 receptor antagonists. J Neurosci 2001;21:303-13.

[15] Gainetdinov RR. Dopamine transporter mutant mice in experiment neuropharmacology. N-S Arch Pharmacol 2008;377:301-13.

[16] Graybiel AM. The basal ganglia and chunking of action repertories. Neurobiol Learn Mem 1998;70:119-36.

[17] Haber SN, Calzavara R. The cortico-basal ganglia integrative network: the role of the thalamus. Brain Res Bull 2009;78:69-74.

[18] Alexander GE, Crutcher MD. Functional architecture of basal ganglia circuits: neural substrates of parallel processing. Trends Neurosci 1990;13:266-71.

[19] Lewis MH, Presti MF, Lewis JB, Turner CA. The neurobiology of stereotypy I: environmental complexity. In: Mason G, Rushen J, editors. Stereotypic animal behaviour: fundamentals and applications to welfare. Wallingford, England: CABI; 2006. p. 190-226.

[20] Graybiel AM. The basal ganglia. Curr Biol 2000;10:509-11.

[21] Luria AR. Two kinds of motor perseveration in massive injury of the frontal lobes. Brain 1965;88:1-12.

[22] Frith CD, Done DJ. Stereotyped responding by schizophrenic patients on a twochoice guessing task. Psychol Med 1983;13:779-86.

[23] Turner M. Towards an executive dysfunction account of repetitive behaviour in autism. In: Russel J, editor. Autism as an executive disorder. Oxford: Oxford University Press; 1997. p. 57-100.

[24] Gluck JP, Sackett GP. Extinction deficits in socially isolated rhesus monkeys (Macaca mulatta). Dev Psychol 1976;12:173-4

[25] Evenden JL, Robbins TW. Increased response switching, perseveration and perseverative switching following d-amphetamine in the rat. Psychopharmacology 1983;80:67-73.

[26] Mason GJ, Latham NR. Can't stop, won't stop: is stereotypy a reliable animal welfare indicator? Anim Welfare 2004;13:S57-69.

[27] Meyer-Holzapfel M. Abnormal behavior in zoo animals. In: Fox MW, editor. Abnormal behavior in animals. London: Saunders; 1968. p. 476-503.

[28] Powell SB, Newman HA, Pendergast JF, Lewis MH. A rodent model of spontaneous stereotypy: initial characterization of developmental, environmental, and neurobiological factors. Physiol Behav 1999;66:355-63.

[29] Shyne A. Meta-analytic review of the effects of enrichment on stereotypic behavior in zoo mammals. Zoo Biol 2006;25:317-37.

[30] Kennes D, Ödberg FO, Bouquet Y, De Rycke PH. Changes in naloxone and haloperidol effects during the development of captivity-induced jumping stereotypy in bank voles. Eur J Pharmacol 1988;153:19-24.

[31] Turner CA, Yang MC, Lewis MH. Environmental enrichment: effects on stereotyped behavior and regional neuronal metabolic activity. Brain Res 2002;938:15-21.

[32] Turner CA, Lewis MH, King MA. Environmental enrichment: effects on stereotyped behavior and dendritic morphology. Dev Psychobiol 2003;43:20-7.

[33] Turner CA, Lewis MH. Environmental enrichment: effects on stereotyped behavior and neurotrophin levels. Physiol Behav 2003;80:259-66.

[34] Garner JP, Mason GJ. Evidence for a relationship between cage stereotypies and behavioural disinhibition in laboratory rodents. Behav Brain Res 2002;136:83-92.

[35] Garner JP, Mason GJ, Smith R. Stereotypic route-tracing in experimentally caged songbirds correlates with general behavioural disinhibition. Anim Behav 2003;66:711-27.

[36] Garner JP, Meehan CL, Mench JA. Stereotypies in caged parrots, schizophrenia and autism: evidence for a common mechanism. Behav Brain Res 2003;145:125-34

[37] Vickery SS, Mason GJ. Behavioral persistence in captive bears: implications for reintroduction. Ursus 2003;14:35-43.

[38] Hemmings A, McBride SD, Hale CE. Perseverative responding and the aetiology of equine oral stereotypy. Appl Anim Behav Sci 2007;104:143-50.

39] Tanimura Y, Yang MG, Lewis MH. Procedural learning and cognitive flexibility in a mouse model of restricted, repetitive behaviour. Behav Brain Res 2008;189:250-6.

[40] Latham N, Mason G. Frustration and perseveration in stereotypic captive animals: is a taste of enrichment worse than none at all? Behav Brain Res 2010;211:96-104.

[41] Garner JP, Thogerson CM, Dufour BD, Würbel H, Murray JD, Mench JA. Reversetranslational biomarker validation of abnormal repetitive behaviors in mice: an illustration of the 4P's modeling approach. Behav Brain Res 2011;219:189-96.

[42] Sandson J, Albert ML. Varieties of perseveration. Neuropsychologia 1984;22:715-32.

[43] Gross AN, Engel AKJ, Richter SH, Garner JP, Würbel H. Cage-induced stereotypies in female ICR CD-1 mice do not correlate with recurrent perseveration. Behav Brain Res 2011;216:613-20.

[44] Hauser MD, Perseveration. inhibition and the prefrontal cortex: a new look. Curr Opin Neurobiol 1999;9:214-22.

[45] Robbins TW. The 5-choice serial reaction time task: behavioural pharmacology and functional neurochemistry. Psychopharmacology 2002;163:362-80.

[46] Cabib S. The neurobiology of stereotypy II: the role of stress. In: Mason G, Rushen J, editors. Stereotypic animal behaviour: fundamen- tals and applications to welfare. Wallingford, England: CABI; 2006. p. 227-55.

[47] Swaisgood R, Shepherdson D. Environmental enrichment as a strategy for mitigating stereotypies in zoo animals: a literature review and meta-analysis. In: Mason G, Rushen J, editors. Stereotypic animal behaviour: fundamentals and applications to welfare. Wallingford, England: CABI; 2006. p. 256-85.

[48] Cooper JJ, Ödberg F, Nicol CJ. Limitations on the effectiveness of environmenta improvement in reducing stereotypic behaviour in bank voles (Clethrionomys glareolus). Appl Anim Behav Sci 1996;48:237-48.

[49] Tilly SLC, Dallaire J, Mason GJ. Middle-aged mice with enrichment-resistan stereotypic behaviour show reduced motivation for enrichment. Anim Behav 2010;80:363-73.

[50] Tanimura Y, Vaziri S, Lewis MH. Indirect basal ganglia pathway mediation of repetitive behavior: attenuation by adenosine receptor agonists. Behav Brain Res 2010;210:116-22.

[51] Jones MA, Mason GJ, Pillay N. The wild-caught 22: why wild-caught striped mice seldom stereotype. Anim Behav submitted; Unpublished results.

[52] Zohar AH, LaBuda M, Moschel-Ravid O. Obsessive-compulsive behaviors and cognitive functioning: a study of compulsivity, frame shifting and type A activity patterns in a normal population. Neuropsychiatr Neuropsychol Behav 1995;8:163-7

[53] Mason GJ. Age and context affect the stereotypies of caged mink. Behaviour 1993; 127:191-229.

[54] Hansen SW, Malmkvist J, Palme R, Damgaard BM. Do double cages and access to occupational materials improve the welfare of farmed mink? Anim Welfare 2007; 16:63-76.

[55] Hansen CPB, Jeppesen LL. Short term behavioural consequences of denied access to environmental facilities in mink. Agr Food Sci Finland 2000;9:149-55.

[56] Vinke CM, Houx BB, Van Den Bos R, Spruijt BM. Anticipatory behaviour and stereotypical behaviour in farmed mink (Mustela vison) in the presence absence and after the removal of swimming water. Appl Anim Behav Sc 2006;96:129-42.

[57] Hansen SW, Jeppesen LL. Temperament, stereotypies and anticipatory behaviour as measures of welfare in mink. Appl Anim Behav Sci 2006;99:172-82.

[58] Dunstone N. The mink. London: T. \& A. D. Poyser Ltd.; 1993.

[59] Mason GJ, Cooper J, Clarebrough C. Frustrations of fur-farmed mink. Science 2001;410:35-6.

[60] Frith U.Studies in pattern detection in normal and autistic children II: reproduction and production of color sequences. J Exp Child Psychol 1970;10:120-35.

[61] Frith U. Cognitive mechanisms in autism: experiments with color and tone sequence production. J Autism Child Schiz 1972;2:160-73.

[62] Boucher J. Alternation sequencing behaviour and response to novelty in autistic children. J Child Psychol Psychiatr 1977;18:67-72.

[63] Lyon N, Mejsholm B, Lyon M. Stereotyped responding by schizophrenic outpatients: cross-cultural confirmation of perseverative switching on a two-choice task. J Pyschiatr Res 1986;20:137-50.

[64] Ridley RM, Baker HF, Frith CD, Dowdy J, Crow TJ. Stereotyped responding on a two-choice guessing task by marmosets and humans treated with amphetamine. Psychopharmacology 1988;95:560-4

[65] Peng CK, Buldyrev SV, Havlin S, Simons M, Stanley HE, Goldberger AL. Mosaic organization of DNA nucleotides. Phys Rev E 1994;49:1685-9.

[66] Asher L, Collins LM, Ortiz-Pelaez A, Drewe JA, Nicol CJ, Pfeiffer DU. Recent advances in the analysis of behavioural organization and interpretation as indicators of animal welfare. J R Soc Interface 2009;6:1103-19.

[67] Diamond A. Developmental time course in human infants and infant monkeys, and the neural bases of, inhibitory control in reaching. Ann NY Acad Sci 1991;608:637-76.

[68] Jentsch JD, Redmond Jr DE, Elsworth JD, Taylor JR, Youngren KD, Roth RH. Enduring cognitive deficits and cortical dopamine dysfunction in monkeys after long-term administration of phencyclidine. Science 1997;277:953-5.

[69] Walker SC, Mikheenko YP, Argyle LD, Robbins TW, Roberts AC. Selective prefrontal serotonin depletion impairs acquisition of a detour-reaching task. Eur ] Neurosci 2006;23:3119-23.

[70] Smith BP, Litchfield CA. How well do dingoes, Canis dingo, perform on the detour task? Anim Behav 2010;80:155-62.

[71] Lockman JT. The development of detour ability during infancy. Child Dev 1984;55:482-91.

[72] Darlington RB, Smulders TV. Problems with residual analysis. Anim Behav 2001;62:599-602.

[73] García-Berthou E. On the misuse of residuals in ecology: testing regression residuals vs. the analysis of covariance. J Anim Ecol 2001;70:708-11.

[74] Regolin L, Vallortigara G, Zanforlin M. Perceptual and motivational aspects of detour behaviour in young chicks. Anim Behav 1994;47:123-31.

[75] Pongrácz P, Miklósi Á, Kubinyi E, Gurobi K, Topál J, Csányi V. Social learning in dogs: the effect of a human demonstrator on the performance of dogs in a detour task. Anim Behav 2001;62:1109-17.

[76] Wilkinson A, Kuenstner K, Mueller J, Huber L. Social learning in a non-socia reptile (Geochelone carbonaria). Biol Lett 2010;23:614-6.

[77] Vlamings PHJM, Hare B, Call J. Reaching around barriers: the performance of the great apes and 3-5-year-old children. Anim Cogn 2010;13: 273-85.

[78] Chapuis N, Thinus-Blanc C, Poucet B. Dissociation of mechanisms involved in dogs' oriented displacements. Q J Exp Psychol-B 1983;35:213-9.

[79] Poucet B, Thinus-Blanc C, Chapuis N. Route planning in cats, in relation to the visibility of the goal. Anim Behav 1983;31:594-9. 
[80] Talpos JC, Wilkinson LS, Robbins TW. A comparison of multiple 5-HT receptors in two tasks measuring impulsivity. Psychopharmacology 2006;20:47-58.

[81] Haber SN. The primate basal ganglia: parallel and integrative networks. J Chem Neuroanat 2003;26:317-30.

[82] Chen JF, Beilstein M, Hu YH, Turner TJ, Moratalla R, Standaert DG, et al. Selective attenuation of psychostimulant-induced behavioral responses in mice lacking $A_{2 A}$ adenosine receptors. Neuroscience 2000;97:195-204.

[83] Robbins TW, Everitt BJ. Functions of dopamine in the dorsal and ventral striatum. Semin Neurosci 1992;4:119-27.

[84] Cardinal RN, Pennicott DR, Sugathapala CL, Robbins TW, Everitt BJ. Impulsive choice induced in rats by lesions of the nucleus accumbens core. Science 2001;292:2499-501.

[85] Perry JL, Stairs DJ, Bardo MT. Impulsive choice and environmental enrichment: effects of D-amphetamine and methylphenidate. Behav Brain Res 2008;193:48-54
[86] Zakharova E, Miller J, Unterwald E, Wade D, Izenwasser S. Social and physical environment alter cocaine conditioned place preference and dopaminergic markers in adolescent male rats. Neuroscience 2009;163. 890-7.

[87] McBride SD, Hemmings A. Altered mesoaccumbens and nigro-striatal dopamine physiology is associated with stereotypy development in a nonrodent species. Behav Brain Res 2005;159:113-8.

[88] Terlouw EMC, Lawrence AB, Illius AW. Relationship between amphetamine and environmentally induced stereotypies in pigs. Pharmacol Biochem Behav 1992;43:347-55.

[89] Parkinson JA, Olmstead MC, Burns LH, Robbins TW, Everitt BJ. Dissociation in effects of lesions of the nucleus accumbens core on appetitive Pavlovian approach behavior and the potentiation of conditioned reinforcement and locomotor activity by D-amphetamine. J Neurosci 1999;19: 2401-11. 